\title{
Simulating the impact of dust cooling on the statistical properties of the intracluster medium
}

\author{
Etienne Pointecouteau \\ Université de Toulouse/CNRS, CESR, 9 av. du Colonel Roche, 31400 Toulouse, \\ France \\ Antonio da Silva \\ CAUP, Rua das Estrelas, 4150-762 Porto, Portugal \\ Institut d'Astrophysique Spatiale, Bat 121, Universite Paris Sud, 91405 Orsay, \\ France \\ Andrea Catalano \\ Universit Paris Diderot, APC, 10, rue Alice Domon et Lonie Duquet, 75205 \\ PARIS cedex 13, France
}

Ludovic Montier, Joseph Lanoux, Mauro Roncarelli, Martin Giard

Université de Toulouse/CNRS, CESR, 9 av. du Colonel Roche, 31400 Toulouse, France

\begin{abstract}
From the first stages of star and galaxy formation, non-gravitational processes such as ram pressure stripping, SNs, galactic winds, AGNs, galaxy-galaxy mergers, etc... lead to the enrichment of the IGM in stars, metals as well as dust, via the ejection of galactic material into the IGM. We know now that these processes shape, side by side with gravitation, the formation and the evolution of structures. We present here hydrodynamic simulations of structure formation implementing the effect of the cooling by dust on large scale structure formation. We focus on the scale of galaxy clusters and study the statistical properties of clusters. Here we present our results on the $T_{X}-M$ and the $L_{X}-M$ scaling relations which exhibit changes on both the slope and normalization when adding cooling by dust to the standard radiative cooling model. For example, the normalization of the $T_{X}-M$ relation changes only by a maximum of $2 \%$ at $M=10^{14} \mathrm{M}_{\odot}$ whereas the normalization of the $L_{X}-T_{X}$ changes by as much as $10 \%$ at $T_{X}=1 \mathrm{keV}$ for models that including dust cooling. Our study shows that the dust is an added non-gravitational process that contributes shaping the thermodynamical state of the hot ICM gas.
\end{abstract}

Preprint submitted to Elsevier

8 November 2018 
Key words: Astrophysics, numerical simulations, galaxy clusters

\section{Introduction}

The high metallicity observed in the intra-cluster medium (ICM hereafter - see for instance Borgani et al. (2008), for recent works) is understood within analytical models and numerical simulations as a consequence of the various gravitational (galaxy-galaxy mergers, galaxy interactions, ram pressure stripping) and non-gravitational (SN powered galactic winds, AGN outbursts, starburts winds) processes at play in the ICM. These processes drive the formation and the evolution of galaxies within their environment (see for instance works by Kapferer et al. (2006); Domainko et al. (2006); Moll et al. (2007). Moreover, it is obvious that the process of tearing material from galaxies does not discriminate between metals, gas, stars and dust. All are injected in the ICM/IGM further on leading to the metal enrichment of the surrounding medium, and possibly to its dust enrichment. To date the searches for a diffuse dust component within the ICM have not been significantly successful. Together with their study of the reddening of background galaxies towards clusters, Muller et al. (2008) gathered the existing constraints on the presence of dust in the ICM and the derived constraints in terms of extinction or dust-to-gas mass ratio. Montier and Giard (2005) obtained a clear statistical detection for the total dust emission in clusters (from member galaxies and ICM) by using the stacking of the IRAS data (at 60 and $100 \mu \mathrm{m}$ near the peak of dust emission) at the location of more than 11000 clusters and groups. In an extension of this work, Giard et al. (2008) have investigated for the first time the evolution of the total IR bolometric luminosity of clusters with redshift. Comparing to the expected IR luminosity from the integrated SFR in cluster galaxies and considering the low end of current constraints for the dust-to-gas mass ratio in the ICM (Chelouche et al., 2007), Giard et al. (2008) concluded that ICM dust could represent $2 \%$ of the bolometric IR luminosity of clusters. On the other hand, from a theoretical point of view the effect of dust on a ICM/IGMtype thermalized plasma has been formalised by Montier and Giard (2004). These authors have computed the cooling function of dust within an optically thin hot thermal plasma taking into account the energetic budget for dust. In view of their results, dust thus comes within the ICM/IGM, as an added non-gravitational process that may influence the formation and evolution of large scale structures in a non-negligible way.

In order to address this problem, we have put into place the first hydrodynamic

Email address: pointeco@cesr.fr (Etienne Pointecouteau). 

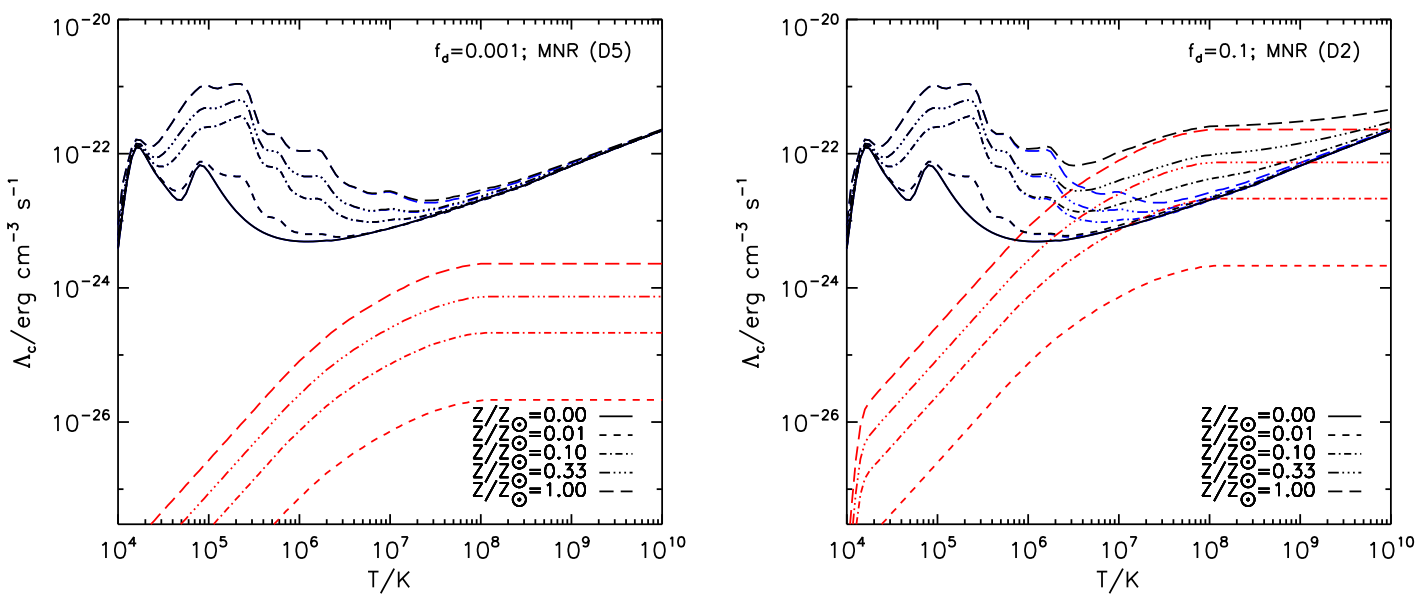

Fig. 1. Total cooling (radiative + dust) functions implemented in the numerical simulations (black lines). The radiative and dust cooling functions alone are respectively shown in blue and red. The figure illustrate the dependency of the dust cooling efficiency as a function of the metallicity if the IGM (red curves on both panels) and as a function of the dust-to-gas mass ratio within the ICM (left and right panels).

$N$-body numerical simulations of hierarchical structure formation implementing the effect of dust cooling according to the dust nature and abundance. In this proceedings paper, we present original preliminary results of this study focussing on the properties of clusters of galaxies at redshift zero.

\section{Cooling the ICM with dust}

Montier and Giard (2004) have shown that in the temperature range of galaxy clusters, dust can be a cooling agent of the ICM, which can compete efficiently with radiative cooling. The strength of cooling by dust depends on dust grain properties and more specifically on dust abundance and dust grain sizes. The more abundant the dust and the smaller the grains, the higher the efficiency of the cooling by dust is.

- The dust abundance, i.e the dust-to-gas mass ratio, is a function of the relative abundance of dust with respect to the solar vicinity dust abundance (i.e $Z_{d, \odot}$ ) and of the metallicity of the ICM (i.e $\left.Z / Z_{\odot}\right): Z_{d}=M_{d u s t} / M_{\text {gas }}=$ $f_{d}\left(Z / Z_{\odot}\right) Z_{d, \odot}$. We investigate three values for $f_{d}: 0.001,0.01$ and 0.1 . The corresponding dust-to-gas mass ratios bracket roughly the current theoretical and observational constraints on dust abundance in the ICM/IGM, i.e 10. ${ }^{-5}$ and $10^{-3}$ (Popescu et al., 2000; Aguirre et al., 2001; Chelouche et al., 2007; Muller et al., 2008; Giard et al., 2008).

- At a given metallicity, the most relevant parameter for the cooling function by dust is the grain size. Indeed, the smaller the grains, the higher the cooling power of the dust is. We chose to test three types of size distribution: 


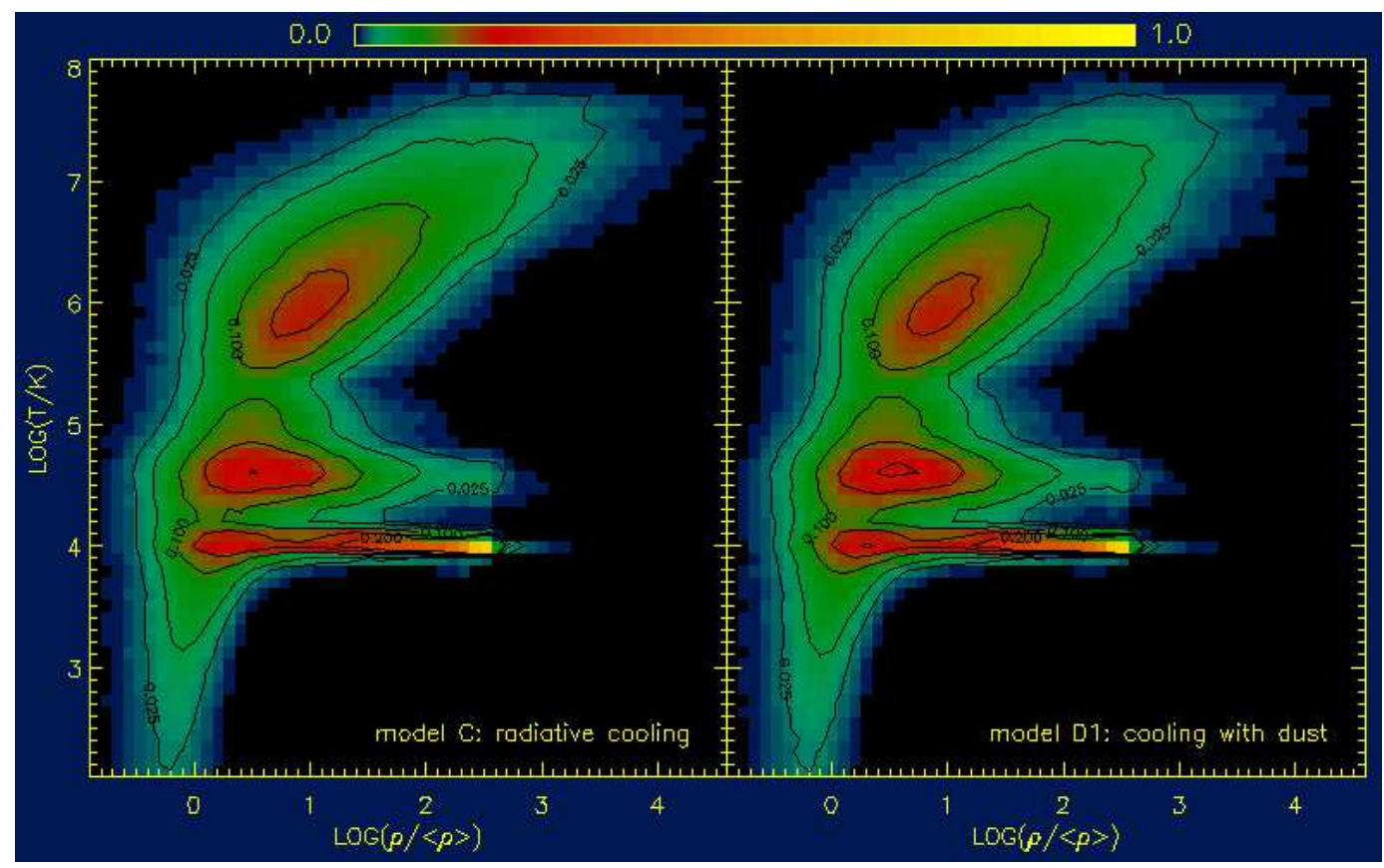

Fig. 2. Density-Temperature phase space diagram of the radiative cooling simulation (left) and a case of radiative+dust cooling simulation. The separation of the different phases (ICM, IGM, cool gas) is illustrated by the dashed-yellow lines. The overlaid contours are isoprobability contours of $0.025,0.05,0.1,0.15$ and 0.2 .

two fixed grain sizes with $a=10^{-3} \mu \mathrm{m}$ and $a=0.5 \mu \mathrm{m}$, respectively labelled small and big. The third assumes for the IGM dust grains a distribution in sizes as defined by Mathis et al. (1977) for the galactic dust: $N(a) \propto a^{-3.5}$ within the size interval of $[0.001,0.5] \mu \mathrm{m}$. It is further referred as the 'MNR' distribution.

Thus in our simulations, once the metallicity is known, $a$ and $f_{d}$ are the only two parameters driving the dust cooling rate (i.e $\Lambda(a, Z)=\Lambda\left(a, f_{d}\right)$ ). Fig. 1 illustrates the dependency of the dust cooling function with respect to the metallicity and dust abundances for a MNR dust grain size distribution.

\section{Simulation of structure formation with dust cooling}

\subsection{Hydrodynamic $\mathrm{N}$-body simulations}

We have run a set of seven numerical simulations using the public code package Hydra, an adaptive particle-particle/particle-mesh $\left(\mathrm{AP}^{3} \mathrm{M}\right)$, gravity solver with smoothed particle hydrodynamics (SPH) (Couchman et al., 1995; Couchman, 1991; Pearce and Couchman, 1997). The global gas metallicity is assumed to evolve with time as $Z=0.3\left(t / t_{0}\right) Z_{\odot}$, where $Z_{\odot}$ is the solar metallicity and $t / t_{0}$ is the age of the universe in units of the present time. All simulations are issued from the same initial condition snapshot, at $z=49$. The 
initial density field was constructed, using $N=4$, 096, 000 particles of baryonic and dark matter, perturbed from a regular grid of fixed comoving size $L=100 h^{-1} \mathrm{Mpc}$. We assumed a $\Lambda$-CDM cosmology with parameters, $\Omega=0.3$, $\Omega_{\Lambda}=0.7, \Omega_{b}=0.0486, h=0.7$. The amplitude of the matter power spectrum was normalised using $\sigma_{8}=0.9$. With this choice of parameters, the dark matter and baryon particle masses are $2.1 \times 10^{10} h^{-1} \mathrm{M}_{\odot}$ and $2.6 \times 10^{9} h^{-1} \mathrm{M}_{\odot}$ respectively. The gravitational softening in physical coordinates was $25 h^{-1} \mathrm{kpc}$ below $z=1$ and above this redshift scaled as $50(1+z)^{-1} h^{-1} \mathrm{kpc}$. Gas in the simulations is allowed to cool using the total table presented in Fig 1. At a given time step, gas particles with overdensities (relative to the critical density) larger than $10^{4}$, and temperatures below $1.2 \times 10^{4} \mathrm{~K}$ are converted into collisionless baryonic matter and no longer participate in the gas dynamical processes.

\subsection{Phase space and cluster maps}

On Fig. 2 we present the phase space diagram for the whole box at $z=0$ for the radiative cooling (left panel) and the radiative-dust cooling model with $a=0.001 \mu \mathrm{m}$ and $f_{d}=0.1$ (right panel - model D1 in Table 1 ).

The displayed quantity is the probability distribution function of gas particles (computed as the number of particles in logarithmic bins of size 0.1 dex divided by the total number of particles) as a function of temperature and overdensity. The colour scale is linear, as represented on the top of the figure. The features in the diagrams reflect the gas physical effects included in the simulations: gravitational heating, adiabatic expansion, radiative cooling and cooling by dust (the latter effect is only present on the right panel). Note that our simulations do not include energy feedback nor photoinization-heating due to a UV background. The absence of the later effect causes the low density, low temperature, gas to cool adiabatically without being regulated by photoionization heating. For $T>10^{4} \mathrm{~K}$ gas cools more efficiently according to the cooling rates of Fig. 1. At high overdensities $\log (\rho /<\rho>) \gtrsim 2.5$ gas is mainly distributed in bound objects such as galaxy groups and clusters with high temperatures. The gas in these regions cools rapidly due to the absence of energy feedback. The inclined V-shape (in black) on the far right end side of the diagrams is a region of fast cooling, where the gas cannot remain over a Hubble time. In both panels, the two upper peaks at intermediate overdensities reflect the shape of the cooling functions in Fig. 1. The contribution of cooling due to metals $\left(Z / Z_{\odot}=0.3\right.$ at $z=0$ in our simulations) increases the cooling efficiency at $T=10^{6} \mathrm{~K}$ as compared to a gas with primordial composition. The absence of non-gravitational heating in our simulations also enhances the appearance of these two upper peaks in the phase space diagram. The horizontal peak at $T \simeq 10^{4} \mathrm{~K}$ arises because in our simulations the gas below this temperature can only cool adiabatically. At high overdensities, gas that cools below $10^{4} \mathrm{~K}$ is considered collisionless baryonic matter and it is 


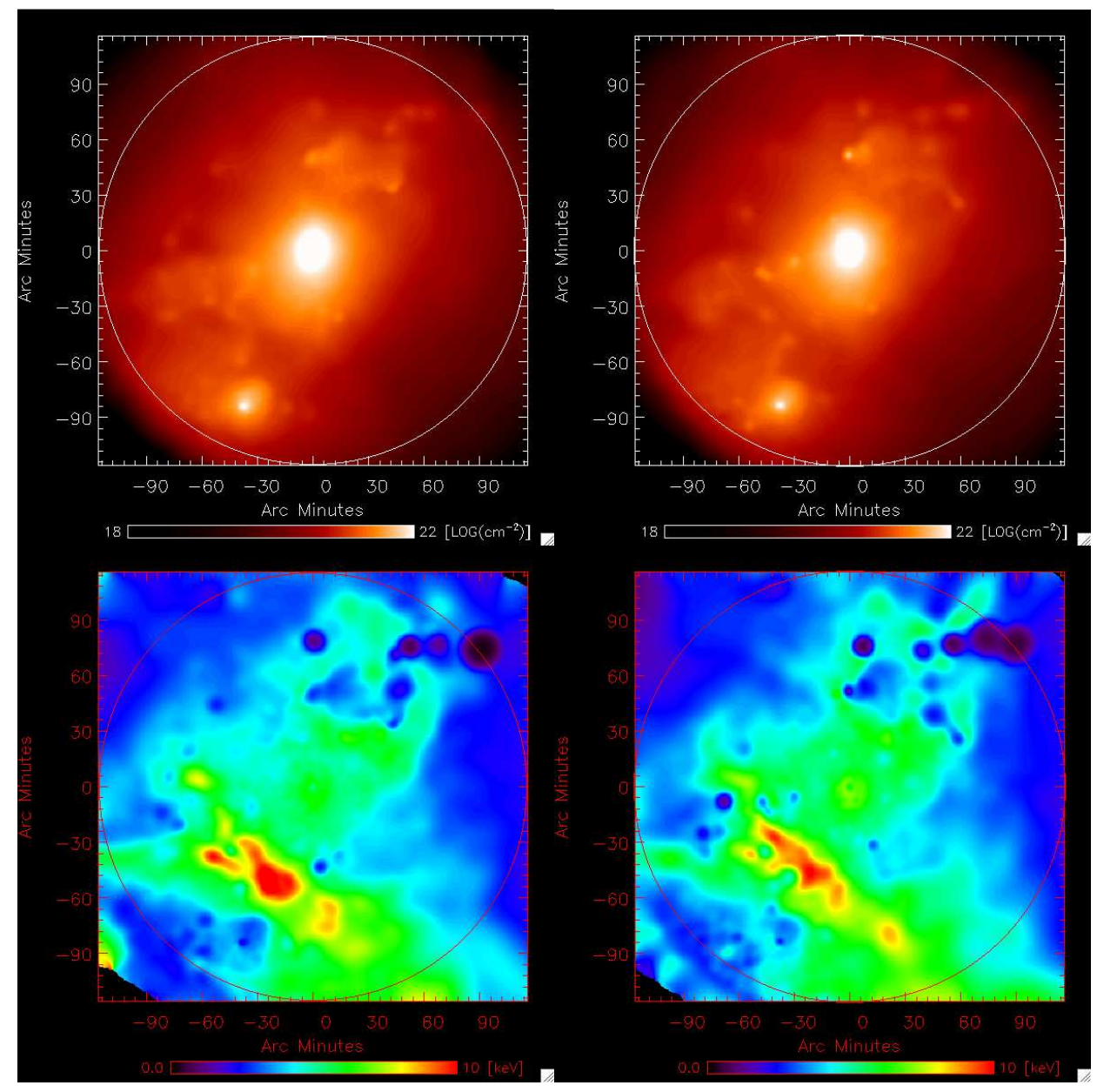

Fig. 3. Projected density (top pane) and temperature (bottom) maps for one of the most massive clusters found in the simulations. The radiative case is presented on the left hand side, whilst a model implementing radiative+dust cooling is presented on the right hand side of the figure. Density and temperature maps are respectively in logarithmic and linear scales. The overlaid circle's radius is the virial radius of the cluster. The $\mathrm{X}$ and $\mathrm{Y}$ axis are given in arcmin considering a projection of the clusrter positionned at $z=0.03$ (in this case the spatial scales are just indicative, but meaningless).

converted into galaxy fragments/star forming material.

The differences between the two diagrams are not striking. However, the concentration of gas in the cool and low-density phase of the D1 model is higher than in the purely gas cooling case (model C). For the ICM gas, the phase space distributions are also different when looked in detail. More particles are present in the form of cold gas (i.e bellow the horizontal yellow lines) in the radiative+dust cooling case, as a direct consequence of the extra cooling from the dust component. As a consequence, the hot and less dense gas distribution (typically located in the clusters core, i.e. top-right parts of the diagrams) is more spread towards slightly less hot and dense gas in the radiative+dust 
Table 1

Parameters of the simulated models presented on Fig 4.

\begin{tabular}{lccc}
\hline \hline Run & Physics & $f_{d}$ & Grain size \\
\hline \hline C & cooling (no dust) & - & - \\
\hline D1 & cooling with dust & 0.100 & small \\
D2 & cooling with dust & 0.100 & MRN \\
D4 & cooling with dust & 0.010 & MRN \\
\hline \hline
\end{tabular}

cooling case. This is due to a well-known effect related with the condensation of gas that is converted into collisionless star forming material in the simulations. The removal of cold gas from the hot phase simply leads to an increase of the mean local temperature of the remaining gas.

For the same models we display in Fig. 3 the projected density and temperature maps for one of the most massive cluster found in our simulations. As for the phase space, the changes in features on the density maps are not obvious. However, the locus of high density seems brighter in the radiative+dust cooling case, e.g the cluster centre is brighter and more peaked. The temperature maps present more visible differences between the dust/no-dust case. Some hot areas are enhanced or have appeared when adding dust cooling. This is also a consequence of the aforementioned (counter intuitive) effect of additional cooling (e.g due to dust) actually contributes to raise the temperature of the remaining ICM gas.

\section{Cluster scaling relations}

\section{1 $T_{X}-M$ and $L_{X}-M$ at redshift zero}

In fact, to be able to properly quantify the effect of the cooling by dust in the ICM, we have investigated the statistical properties of the cluster populations forming in our simulations. In each simulation we selected halos with a total mass larger than $5 \times 10^{13} \mathrm{M} \odot$. We ended up with a minimum of 60 clusters in each simulations at $z=0$. Here we illustrate our study with two scaling relations given at $z=0$ : the (emission weighted) temperature-mass relation, $T_{X}-M$, and the X-ray bolometric luminosity-mass relation, $L_{X}-M$. The two relations are presented on Fig. 4 for the radiative cooling case, and three radiative+dust models described in Table 1 .

The $T_{X}-M$ relation is the less disturbed of the two. Results for model D4 is almost compatible with the radiative case. Model D2 and D1 illustrate the effects of the dust grain size distribution and the dust abundance. 

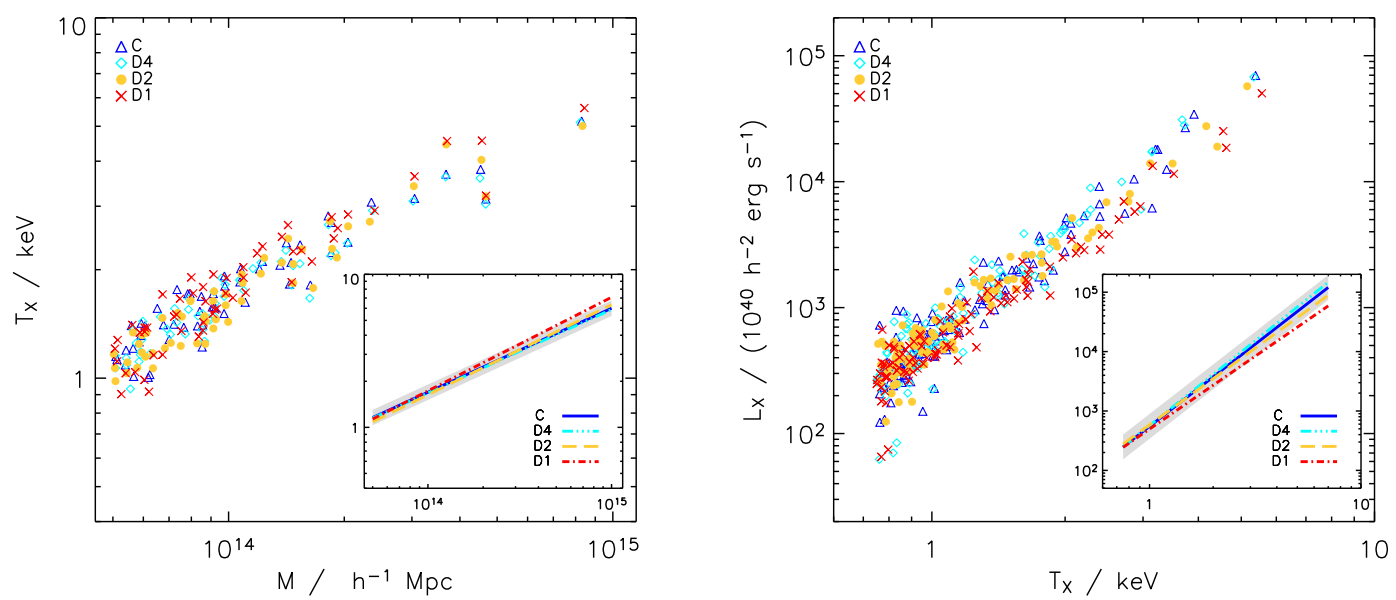

Fig. 4. (left) Emission weighted temperature-mass relation, $T_{X}-M$, (right) X-ray bolometric luminosity-mass relation, $L_{X}-M$, at redshift zero. Symbols and best fit lines are color-coded for each presented model (see Table 1). The grey shaded area corresponds to the $\pm 1 \sigma$ dispersion for the radiative model (i.e model $\mathrm{C}$ ).

These small variations are in fact understandable because the temperature of clusters is essentially dominated by gravity and because the total cluster mass (that is dominated by the dark mater component) is in practice unaffected by the extra condensation of a fraction of the cluster gas mass. However small, we note that the emission-weighted vs mass scaling is more sensitive to dust than the mass-weighted vs mass relation studied in da Silva et al. (2009). This confirms expectations, because $T_{X}$ gives more weighting to gas with stronger $\mathrm{X}$-ray emission, i.e gas with high density and temperature locate in the cluster central regions. As a consequence the $T_{X}-M$ scaling not only exhibits larger changes of amplitude but also larger differences in the slopes because of the relative impact of dust on $T_{X}$ for different mass systems. Altogether the variation in normalisation are less than $\simeq 2 \%$ over the 3 presented models at $M=10^{14} \mathrm{M}_{\odot}$.

On the other hand the $L_{X}-M$ relation is typically a baryon driven scaling law. Which means that it is very sensitive to the physics of baryons at play in the ICM. Again, going from model D4 to D1 via D2, one can witness the effect of the grain size (as for D1 and D2 the abundances are the same), and then the abundance effect from model D2 to D4. Both the abundance and the grain size distribution have similar amplitude, and the overall changes in normalisation at $1 \mathrm{keV}$ goes up by $\sim 10 \%$. It is also striking how the dust cooling efficiency increases with the ICM temperature. Taking into account the range of investigated temperatures, this is perfectly consistent with the dust cooling function implemented in the simulations, thus with the study of Montier and Giard (2004) that shows this discriminating effect with the temperature.

Also, we have pointed out that these changes induced by dust cooling on the 
normalisation as well as the slope of the scaling relations, are strongly linked to the physical properties of dust. We have illustrated that the effect of the dust grain size is as efficient as the effect of the abundance of dust grains populating the ICM.

\subsection{Limitations of the model}

It is obvious that our present implementation of dust cooling in simulations has intrinsic limitations and should be considered as a first ("zero order") approach to the problem, see da Silva et al. (2009). Indeed, with our implentation of dust in our simulations, we directly linked the presence (thus abundance) of dust with the presnce of metals (i.e we put dust wherever there are metals in the simulations). However, dust and metals are not sensitive to the same physical processes, and if the metals are not destroyed, the dust grains are sputtered. From Draine and Salpeter (1979) and Montier and Giard (2004), for a hot thermal plasma, i.e $10^{6}<T<10^{9} \mathrm{~K}$, and densities met in the core of dense clusters, i.e $n_{H} \sim 10^{-3} \mathrm{~cm}^{-3}$, and grain sizes ranging from 0.001 and $0.5 \mu \mathrm{m}$, dust grains have lifetimes of $10^{6}<t_{\text {dust }}<10^{9} \mathrm{yr}$. The overall efficiency of cooling by dust in the ICM/IGM is thus strongly linked to the physical processes of dust injection. Therefore, beside the cooling function of dust, with which we have performed a fully self-consistent implementation of the effect of dust as a cooling vector of the ICM/IGM, we did not performed stricto senso a physical implementation, as we do not deal with the physics of the dust creation and dust destruction processes. This would be the next step in our study.

Also, we directly correlated the abundance of dust with metallicity. The evolution law we chose for the metallicity does underestimate the metallicity at high redshift. Indeed, $Z=0.3\left(t / t_{0}\right) Z_{\odot}$ normalised at $0.3 Z_{\odot}$ at $z=0$ gives $\sim 0.2$ at $z=0.5$ and $\sim 0.1$ at $z=1$. However, observations as well as theoretical studies on the metal enrichment of the ICM have indicate high values of the metalicity, i.e $Z \sim 0.3 Z_{\odot}$, up to redshift above 1 (Cora et al., 2008; Borgani et al., 2008). An early enrichment of the ICM/IGM with dust, then sputtered, could (partly) explain these high values of the metallicity. Therefore, by underestimating the metallicity at high redshifts, we may have underestimated the amount of dust injected in the ICM at high redshifts, and thus the efficiency of dust cooling when integrated from an early epoch down to redshift zero

They are probably other limitations to our results, such as the local thermal equilibrium hypothesis underlied in the computation of Montier and Giard (2004) cooling function (following a non-equilibrium hypothesis (Dwek et al., 1990)), that may lead to difference in the cooling curves, the used theoretical setup for the dust emission within an optically thin hot thermal plasma is well suited to our purpose of assessing the influence of dust on the galaxy clusters properties. 


\section{Conclusion}

With our current "zero" order implementation of the effect of dust cooling, we have demonstrated the actual effect of the presence of dust within the IGM/ICM as a non-gravitationnal process that plays a role on the physical properties of baryons within large scale structures. Deeper studies including other scaling laws such as the entropy-mass, $S-M$, and the integrated Comptonisation parameter-mass, $Y-M$, relations are presented in da Silva et al. (2009).

Acknowledgements: We thanks the organisers of the session E14 "The interplay between the Interstellar and Intergalactic Media from High Redshifts to Present" of the 3rth COSPAR assembly, D. Wang and M. Shull, during which the this work was presented. We are also grateful to the two anonymous referees for their fruitful commnents on our manuscript. MR, LM and EP are supported by grant ANR-06-JCJC-0141. AdS was supported by Fundação Ciência Tecnologia (FCT) Portugal under contracts SFRH/BPD/20583/2004 and CIENCIA $200 \%$.

\section{References}

Aguirre, A., Hernquist, L., Schaye, J., Katz, N., Weinberg, D. H., Gardner, J., Nov. 2001. Metal Enrichment of the Intergalactic Medium in Cosmological Simulations. ApJ561, 521-549.

Borgani, S., Diaferio, A., Dolag, K., Schindler, S., Feb. 2008. Thermodynamical Properties of the ICM from Hydrodynamical Simulations. Space Science Reviews 134, 269-293.

Chelouche, D., Koester, B. P., Bowen, D. V., Dec. 2007. The Dust Content of Galaxy Clusters. ApJ (Lett.)671, L97-L100.

Cora, S. A., Tornatore, L., Tozzi, P., Dolag, K., May 2008. On the dynamical origin of the ICM metallicity evolution. MNRAS386, 96-104.

Couchman, H. M. P., Feb. 1991. Mesh-refined P3M - A fast adaptive N-body algorithm. ApJ (Lett.)368, L23-L26.

Couchman, H. M. P., Thomas, P. A., Pearce, F. R., Oct. 1995. Hydra: an Adaptive-Mesh Implementation of P 3M-SPH. ApJ452, 797-+.

da Silva, A. C., Catalano, A., Montier, L., Pointecouteau, E., Lanoux, J., Giard, M., May 2009. The impact of dust on the scaling properties of galaxy clusters. MNRAS, 685-+.

Domainko, W., Mair, M., Kapferer, W., van Kampen, E., Kronberger, T., Schindler, S., Kimeswenger, S., Ruffert, M., Mangete, O. E., Jun. 2006. Enrichment of the ICM of galaxy clusters due to ram-pressure stripping. A\&A452, 795-802 
Draine, B. T., Salpeter, E. E., Jul. 1979. On the physics of dust grains in hot gas. ApJ231, 77-94.

Dwek, E., Rephaeli, Y., Mather, J. C., Feb. 1990. Infrared emission from dust in the Coma cluster of galaxies. ApJ350, 104-109.

Giard, M., Montier, L., Pointecouteau, E., Simmat, E., Nov. 2008. The infrared luminosity of galaxy clusters. A\&A490, 547-554.

Kapferer, W., Ferrari, C., Domainko, W., Mair, M., Kronberger, T., Schindler, S., Kimeswenger, S., van Kampen, E., Breitschwerdt, D., Ruffert, M., Mar. 2006. Simulations of galactic winds and starbursts in galaxy clusters. A\&A447, 827-842

Mathis, J. S., Rumpl, W., Nordsieck, K. H., Oct. 1977. The size distribution of interstellar grains. ApJ217, 425-433.

Moll, R., Schindler, S., Domainko, W., Kapferer, W., Mair, M., van Kampen, E., Kronberger, T., Kimeswenger, S., Ruffert, M., Feb. 2007. Simulations of metal enrichment in galaxy clusters by AGN outflows. A\&A463, 513-518.

Montier, L. A., Giard, M., Apr. 2004. The importance of dust in cooling and heating the InterGalactic Medium. A\&A417, 401-409.

Montier, L. A., Giard, M., Aug. 2005. Dust emission from clusters of galaxies: statistical detection. A\&A439, 35-44.

Muller, S., Wu, S.-Y., Hsieh, B.-C., Gonzalez, R., Loinard, L., Yee, H., Gladders, M., Jan. 2008. Searching for dust in the intracluster medium from reddening of background galaxies.

Pearce, F. R., Couchman, H. M. P., Nov. 1997. Hydra: a parallel adaptive grid code. New Astronomy 2, 411-427.

Popescu, C. C., Tuffs, R. J., Fischera, J., Völk, H., Feb. 2000. On the FIR emission from intracluster dust. A\&A354, 480-496. 